\title{
A RUNAWAY RED SUPERGIANT IN M31*
}

\author{
Kate Anne Evans ${ }^{1,3}$ and Philip Massey ${ }^{1,2}$ \\ ${ }^{1}$ Lowell Observatory, 1400 W Mars Hill Road, Flagstaff, AZ 86001, USA; kevans@ @altech.edu, phil.massey@lowell.edu \\ ${ }^{2}$ Department of Physics and Astronomy, Northern Arizona University, Flagstaff, AZ 86011-6010, USA \\ Received 2015 August 19; accepted 2015 September 22; published 2015 October 14
}

\begin{abstract}
A significant percentage of $\mathrm{OB}$ stars are runaways, so we can expect a similar percentage of their evolved descendants to also be runaways. However, recognizing such stars presents its own set of challenges, as these older, more evolved stars will have drifted farther from their birthplace, and thus their velocities might not be obviously peculiar. Several Galactic red supergiants (RSGs) have been described as likely runaways based on the existence of bow shocks, including Betelgeuse. Here we announce the discovery of a runaway RSG in M31 based on a $300 \mathrm{~km} \mathrm{~s}^{-1}$ discrepancy with M31's kinematics. The star is found about $21^{\prime}$ (4.6 kpc) from the plane of the disk, but this separation is consistent with its velocity and likely age ( $\sim 10 \mathrm{Myr})$. The star, J004330.06+405258.4, is an M2 I, with $M_{V}=-5.7, \log L / L_{\odot}=4.76$, an effective temperature of $3700 \mathrm{~K}$, and an inferred mass of $12-15 M_{\odot}$. The star may be a high-mass analog of the hypervelocity stars, given that its peculiar space velocity is probably $400-450 \mathrm{~km} \mathrm{~s}^{-1}$, comparable to the escape speed from M31's disk.
\end{abstract}

Key words: galaxies: individual (M31) - galaxies: stellar content - Local Group - supergiants

\section{INTRODUCTION}

Massive stars form in giant molecular clouds, creating OB associations (e.g., Lada \& Lada 2003), the members of which share similar space velocities. OB stars that are spatially close to one of these associations, but with discrepant radial velocities, were first noted by Blaauw (1961), who termed these "runaways" and proposed a possible explanation for their origin: if the primary in a binary system sheds a significant fraction of its mass (say, in an SN explosion), then the secondary would be set loose with nearly its orbital velocity. This would cause the star's radial velocity to disagree with that of other members of the association and over time would cause the star to move away from its fellows. A radial velocity study of a large sample of Galactic runaway OB stars by Gies \& Bolton (1986) effectively ruled out this explanation, and instead favored a dynamical evolution explanation, a scenario that has been recently supported by the simulations of Fujii \& Portegies Zwart (2011) and Oh et al. (2015).

Regardless of their origins, a significant percentage of $\mathrm{OB}$ stars are considered runaways (10\%-50\% according to Gies \& Bolton 1986 and references therein). Yet, few evolved massive stars have ever been identified as runaways, presumably because these stars are older, and as a star moves farther away, it begins to lose the context of its birth association. Without that context, unusual velocities become harder to distinguish. Nevertheless, a few red supergiants (RSGs) have been identified as runaways due to the presence of bow shocks (Noriega-Crespo et al. 1997a; Mackey et al. 2012). Bow shocks are known to be present for some OB runaways (NoriegaCrespo et al. 1997b), and so have been considered evidence that a star is a runaway. Galactic RSGs considered to be

\footnotetext{
* Observations reported here were obtained at the MMT Observatory, a joint facility of the University of Arizona and the Smithsonian Institution. This paper uses data products produced by the OIR Telescope Data Center, supported by the Smithsonian Astrophysical Observatory.

${ }^{3}$ Research Experience for Undergraduate participant during the summer of 2015. Current address: California Institute of Technology, 1200 East California Blvd, Pasadena, CA 91125, USA.
}

runaways include Betelgeuse, $\mu$ Cep, and IRC-10414 (Gvaramadze et al. 2014).

While conducting a radial velocity study of RSGs in M31, we discovered that the RSG J004330.06+405258.4 is not only quite isolated, but also possesses a radial velocity $300 \mathrm{~km} \mathrm{~s}^{-1}$ at odds with that expected from its location. We conclude that this is a runaway RSG, the first identified in another galaxy, and the fastest known.

\section{OBSERVATIONS}

J004330.06+405258.4 was one of many M31 RSGs we observed spectroscopically. Our sample came from Massey et al. (2009), who had identified candidate RSGs and foreground stars in M31 using the photometry of the Local Group Galaxy Survey (LGGS; Massey et al. 2006). For very red stars, $V-R$ remains a temperature discernment, but $B-V$ becomes primarily an indicator of surface gravity due to the effects of line blanketing in the $B$ bandpass (Massey et al. 2006). Thus, RSGs can be separated from foreground stars in a $B-V, V-R$ diagram. Candidate RSGs were separated from the candidate foreground stars using the following equation:

$$
B-V=-1.599(V-R)^{2}+4.18(V-R)-0.83 .
$$

We summarized the available photometry for J004330.06 +405258.4 in Table 1. The location of J004330.06+405258.4 in the two-color diagram of our sample is shown in Figure 1, where we have colored the expected RSGs in red and the expected foreground stars in black, based on the above cutoff. The location of our runaway star, J004330.06+405258.4, is very much in the expected RSG area.

The full results of the radial velocity study will be discussed elsewhere, but here we will summarize the data relevant to J004330.06+405258.4. The object was observed using Hectospec (Fabricant et al. 2005), a 300 optical fiber-fed spectrograph on the $6.5 \mathrm{~m}$ MMT telescope. We used the 270 line $\mathrm{mm}^{-1}$ grating, which is blazed at $5000 \AA$, and covers a spectral range from 3650 to $9200 \AA$. The grating provides a dispersion of $1.2 \AA$ pixel $^{-1}$ and a spectral resolution of $6.2 \AA$. 
Table 1

Photometry of J004330.06+405258.4

\begin{tabular}{lrr}
\hline \hline Measurement & $\begin{array}{r}\text { Value } \\
(\text { mags })\end{array}$ & $\begin{array}{r}\text { Error } \\
(\mathrm{mags})\end{array}$ \\
\hline$V^{\mathrm{a}}$ & 19.212 & 0.005 \\
$B-V^{\mathrm{a}}$ & 1.933 & 0.013 \\
$V-R^{\mathrm{a}}$ & 1.077 & 0.006 \\
$K^{\mathrm{b}}$ & 14.525 & 0.087 \\
$J-K^{\mathrm{b}}$ & 1.076 & 0.106 \\
\hline
\end{tabular}

Notes.

${ }^{a}$ From the LGGS (Massey et al. 2006).

${ }^{b}$ From 2MASS (Cutri et al. 2003).

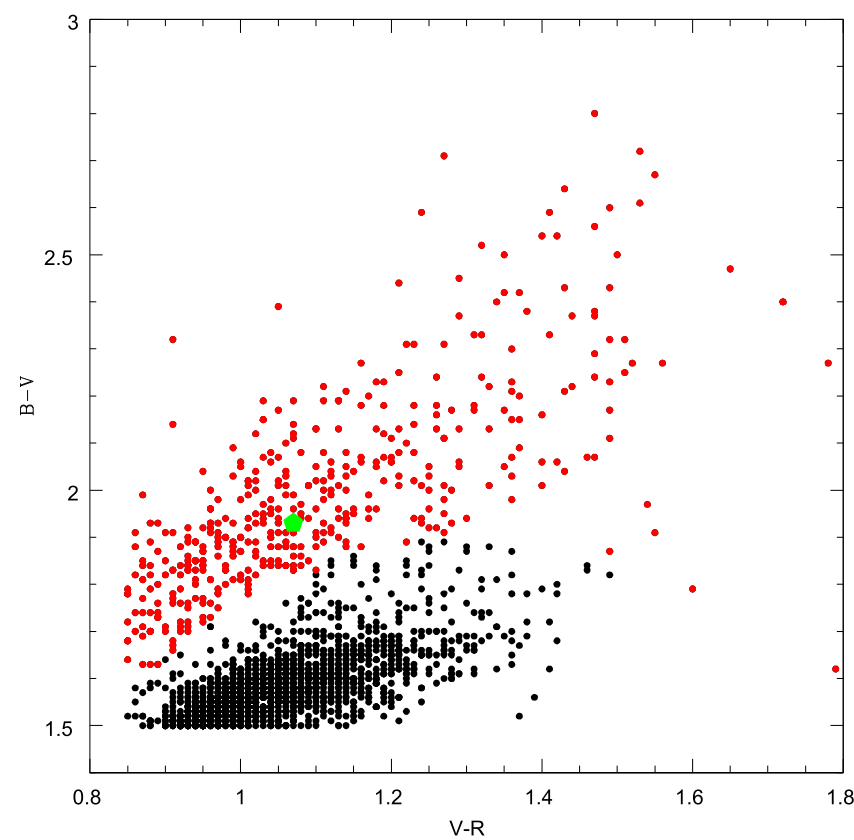

Figure 1. Two-color diagram of red stars observed with Hectospec. The objects in red are suspected red supergiants, and the objects in black are suspected foreground stars. The assumed division between the two is given by Equation (1). J004330.06+405258.6 is represented by the green pentagon.

Some of the fibers were assigned to blank sky to be used for sky subtraction, and calibration included HeNeAr and quartz lamp exposures. Our runaway was observed during four different nights in the fall of 2014 in queue mode for 90 minutes of exposure each, as summarized in Table 2. Following the observations, data were passed through the SAO pipeline, and the wavelength zeropoints were adjusted slightly using night sky lines. The wavelengths were then corrected to a heliocentric reference frame.

As no blocking filter could be used, we expect contamination by second-order blue light at the longer wavelengths of our spectrum. However, at the Ca II triplet lines (8498, 8542, $8662 \AA$ ), which we use for radial velocities, we expect this contamination will be only $\sim 3 \%$, as $B-R \sim 2.9$ (Table 1 ), implying that the overlapping second-order blue at $4250 \AA$ will be roughly a factor of 15 smaller in flux at $8500 \AA$, and the light dispersed by an additional factor of 2 in second order.

Flux calibration was provided by observations of the spectrophotometric standard Feige 34 midway through the semester. The resulting sensitivity curves were kindly provided by Nelson Caldwell. Our experience is that this is typically
Table 2

Radial Velocities and Results of the Model Fitting

\begin{tabular}{|c|c|c|c|c|}
\hline \multirow[t]{2}{*}{ HJD } & \multicolumn{2}{|c|}{$\begin{array}{l}\text { Radial Velo- } \\
\text { city }\left(\mathrm{km} \mathrm{s}^{-1}\right)\end{array}$} & \multicolumn{2}{|c|}{ MARCS Fitting } \\
\hline & $V_{\text {obs }}$ & $\sigma$ & $T_{\text {eff }}(\mathrm{K})$ & $E(B-V)$ \\
\hline 2456926.254 & -625.9 & 1.02 & 3725 & 0.15 \\
\hline 2456982.310 & -633.7 & 1.12 & 3700 & 0.20 \\
\hline 2456987.260 & -623.6 & 1.73 & 3700 & 0.15 \\
\hline 2456989.177 & -632.7 & 1.01 & 3700 & 0.20 \\
\hline Adopted Values & -630 & 1 & 3700 & 0.15 \\
\hline
\end{tabular}

good to $5 \%-10 \%$ in the fluxes relative to wavelengths (i.e., the colors), although occasionally much larger errors may be present (see also Fabricant et al. 2008).

\section{ANALYSIS}

The first step in our larger project is to establish whether the stars selected from the photometry by Massey et al. (2009) were truly RSGs in M31 or merely foreground stars. Except for stars in the NE section of the galaxy, the combination of the $\sim 250 \mathrm{~km} \mathrm{~s}^{-1}$ rotational velocity and $\sim-300 \mathrm{~km} \mathrm{~s}^{-1}$ systemic velocity (de Vaucouleurs et al. 1991) makes it easy to separate foreground Milky Way dwarfs from bona fide M31 members using radial velocities, as shown by Drout et al. (2009) for yellow supergiants (YSGs).

The observed radial velocities were calculated using XCSAO, a cross correlation tool, in IRAF. ${ }^{4}$ For our velocity templates, we used 21 Hectospec spectra of six M31 RSGs for which radial velocities were already known (Massey et al. 2009), and we restricted the cross correlation to the $\mathrm{Ca}$ II triplet lines, which are very strong in RSGs; this avoids using the extremely broad molecular features. We then averaged the result for each spectra, weighting the results in accord with the the internal errors. For stars with more than one observation, the results of each spectra were averaged, with weights assigned by the weighted errors of each spectra. Full details will be given in the larger paper that is in preparation.

We then compared the observed radial velocities with that expected from the star's location in M31 using the simple kinematic model adopted by Drout et al. (2009), which is based on the seminal study by Rubin \& Ford (1970). In general, the expected radial velocity $V_{\exp }$ from circular rotation will be $V_{\text {exp }}=V_{0}+V(R) \sin \xi \cos \theta$, where $V_{0}$ is the systemic radial velocity, $V(R)$ is the rotational velocity at a distance from the galactic center $(R)$ within the plane of the disk, $\xi$ is the angle between the line of sight and the perpendicular to the plane of the galaxy, and $\theta$ is the angle from the semimajor axis. Thus, $\cos \theta$ is $X / R$, where $X$ is the position along the major axis. The gross simplification that $V(R)$ is a constant (which is equivalent to saying that dark matter dominates the kinematics) works remarkably well (Drout et al. 2009; Massey et al. 2009), leaving us with a linear relationship. For consistency with these earlier works, and to simplify using the Rubin \& Ford (1970) data, we have adopted $\xi=77^{\circ}$ and then used $V_{\exp }=-295+241.5(X / R)$. This equation does an excellent job of fitting both the velocities of the $\mathrm{H}$ iI regions measured by Rubin \& Ford (1970) as well as the RSG radial velocities

\footnotetext{
4 IRAF is distributed by the National Optical Astronomy Observatory, which is operated by the Association of Universities for Research in Astronomy (AURA) under a cooperative agreement with the National Science Foundation.
} 


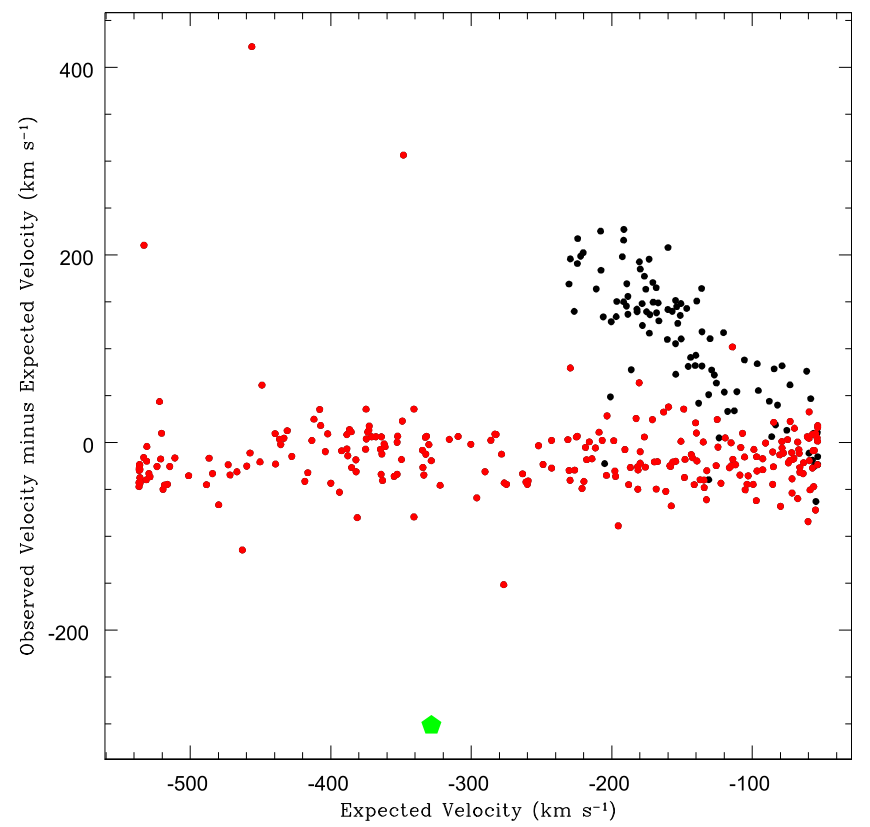

Figure 2. Difference between the observed velocity $\left(V_{\text {obs }}\right)$ and the expected velocity $\left(V_{\text {exp }}\right)$. The stars expected to be RSGs on the basis of their location in the two-color diagram (Figure 1) are shown with red symbols; as expected, these cluster around a velocity difference of 0 . The stars expected to be foreground stars based on their photometry are shown as black symbols, and as as expected these cluster around a line with a slope of -1 in this diagram. The runaway star is noted with a green pentagon.

measured by Massey et al. (2009), as shown in Figure 2 of the latter work.

We compare the velocity difference $\left(V_{\text {obs }}-V_{\text {exp }}\right)$ for each star in Figure 2. The suspected RSGs and the suspected foreground stars split generally well into two distinct groups. The RSGs cluster around a velocity difference of zero, indicating that their observed velocities are about equal to the velocities that we expect based on M31's kinematics. The foreground stars have velocity differences that are about equal to the negative of $V_{\text {exp }}$, as their $V_{\text {obs }}$ values are about zero. It is immediately clear that one star stands out as having a very peculiar negative velocity. This star is J004330.06+405258.4, which we have highlighted with a green pentagon. There are a few other outliers: three purported RSGs that are obviously foreground stars (due to being marginal in the two-color diagram), and several stars whose photometry was compromised by crowding and/or whose spectra were poor. These will be discussed in more detail in our larger paper.

The radial velocities of $\mathrm{J} 004330.06+405258.4$ are given in Table 2, and we can see that the four measurements are quite consistent. Furthermore, the spectra of this star are well exposed, and the triplet lines readily measured; see Figure 4. The observed radial velocity of J004330.06+405258.4 is -630 $\mathrm{km} \mathrm{s}^{-1}$, while its expected radial velocity is $-328 \mathrm{~km} \mathrm{~s}^{-1}$, leading to $\mathrm{a}-302 \mathrm{~km} \mathrm{~s}^{-1}$ peculiar radial velocity.

Consistent with its peculiar radial velocity, J004330.06 +405258.4 is also very well separated spatially from other massive stars, as shown in Figure 3 from the LGGS. We measure a separation of about $21^{\prime}(4.6 \mathrm{kpc})$ from the major axis. Is this separation reasonable? Assuming an age of $10 \mathrm{Myr}$ and a tangental velocity similar to the peculiar component of its radial velocity (i.e., $-300 \mathrm{~km} \mathrm{~s}^{-1}$ ), we can expect $\mathrm{J} 004330.06$

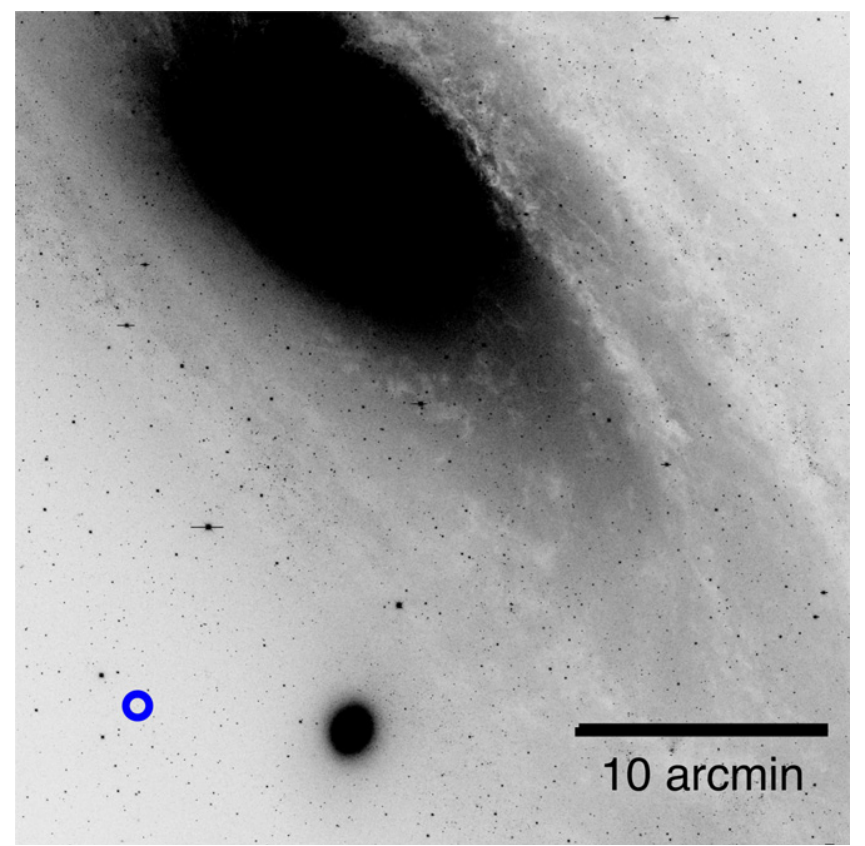

Figure 3. Location of J004330.06+405258.4 in M31. Here J004330.06 +405258.4 is highlighted with a blue circle. The distance from the runaway to the semimajor axis is about $21^{\prime}(4.6 \mathrm{kpc})$. This image was taken in the $V$-band as part of the LGGS. North is up and east is to the left.

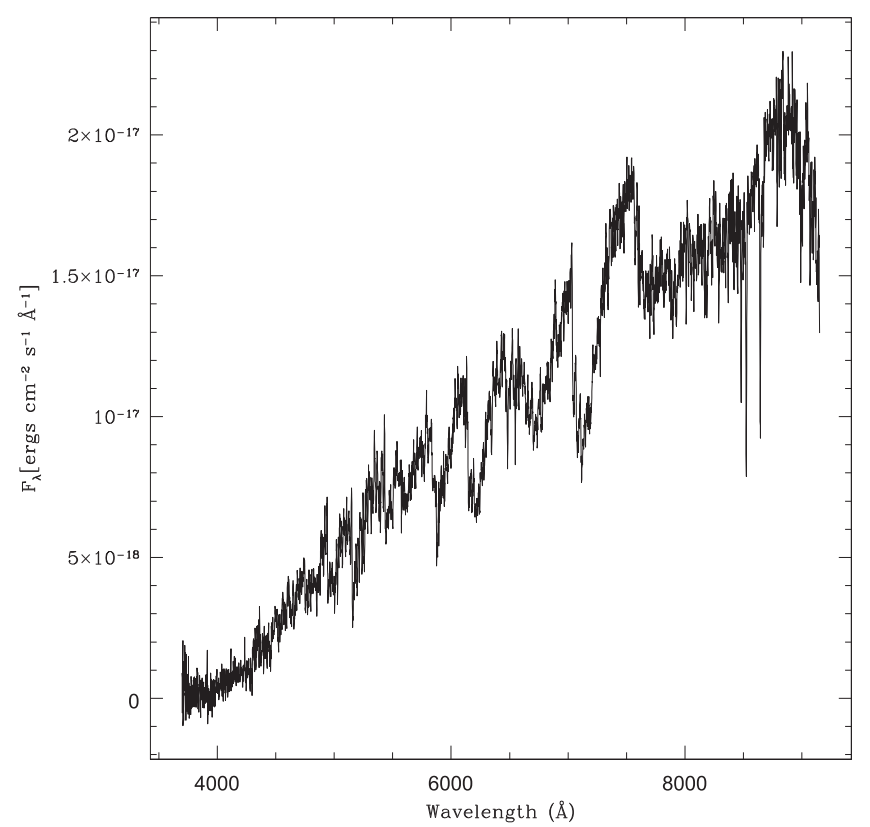

Figure 4. Spectrum of J004330.06+405258.4. The Ca II triplet used for the radial velocities are clearly visible at $8498-8662 \AA$.

+405258.4 to have traveled $\sim 3 \mathrm{kpc}$ from its birthplace, an extremely good match to what we observe. ${ }^{5}$

\subsection{Physical Properties of the Runaway}

We assigned a spectral type of M2 I to J004330.06 +405258.4 . M-type subclasses are determined primarily by

\footnotetext{
5 It takes $11 \mathrm{Myr}$ for a $15 M_{\odot}$ star to become core He-burning, and $15 \mathrm{Myr}$ for a $12 M_{\odot}$ star according to the Geneva evolutionary models (Ekström et al. 2012).
} 


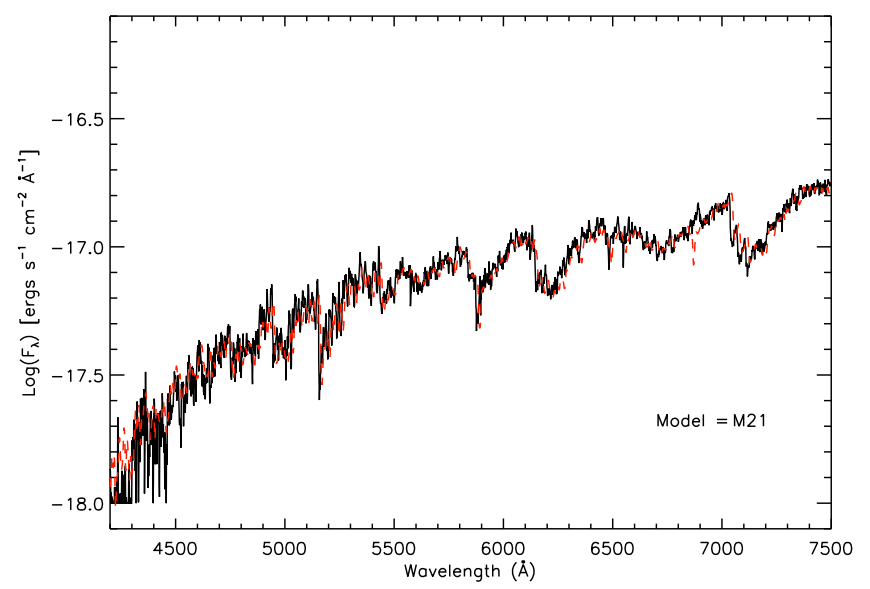

Figure 5. Comparison with an M2 I Spectral Standard. A spectrum of J004330.06+405258.4 (black) is compared with a spectrum of $\mathrm{BD}+59^{\circ} 38$ (red). The latter was classified as M2 I by Levesque et al. (2005).

the depth of the TiO bands. Rather than normalize the spectra (a problematic issue for very red stars where there are only "pseudo" continua), we instead compared the spectra of J004330.06+4052858.4 to stars that had been previously classified by Levesque et al. (2005) digitally in log space. In Figure 5 we show that the spectrum is well matched to that of the $\mathrm{M} 2 \mathrm{I}$ star $\mathrm{BD}+59^{\circ} 38$.

We can determine the effective temperature $T_{\text {eff }}$ of J004330.06+405258.4 using the MARCS stellar models (Gustafsson et al. 2008), following the same procedure as in Massey et al. (2009). We adopted a $\log g$ of 0.0 and compared the spectral features (primarily the depths of the molecular bands) to those of models of different effective temperatures, adjusting the color excess as needed. A "good" fit was determined using the $\mathrm{TiO}$ bands between about 6000 and $7000 \AA$. An example is shown in Figure 6. The results of these fits are shown in Table 2. The temperature of $3700 \mathrm{~K}$ can be compared to the $3675 \mathrm{~K}$ temperature found by Massey et al. (2009) as the median of M2 I stars in M31 using the same super-solar metallicity models.

With this value for the effective temperature we can now determine the bolometric luminosity. We measured $E(B-V)$ values of $0.15-0.20$ from our fitting. This amount of reddening is consistent with the typical 0.13 value found from OB stars by Massey et al. (2007), but given the star's location far from any star-forming region, is probably indicative of some circumstellar component, as is commonly found with RSGs (Massey et al. 2005). Adopting $A_{V}=3.1 \times 0.15=0.46$ and a distance of $760 \mathrm{kpc}$ (from van den Bergh 2000), we find an absolute visual magnitude $M_{V}=-5.65$. The bolometric correction at $V$ for the $3700 \mathrm{~K}$ MARCS model is -1.51 , giving us a bolometric magnitude $M_{\text {bol }}=-7.16$, or $\log L / L_{\odot}$ of 4.76 . Alternatively, we can use the 2MASS photometry (given in Table 1) to find the bolometric luminosity. We expect $A_{K} \sim 0.112 \times A_{V}$ (Schlegel et al. 1998) or 0.05. Thus, $M_{K}=-9.93$. The bolometric correction at $K$ for a star of $3700 \mathrm{~K}$ is +2.76 according to the MARCS models. Thus from the K-band, we derive $M_{\mathrm{bol}}=-7.17$, in near perfect agreement with the value derived from $V$. A comparison with the evolutionary tracks of Ekström et al. (2012) gives an inferred mass of J004330.06 +405258.4 of $12-15 M_{\odot}$.

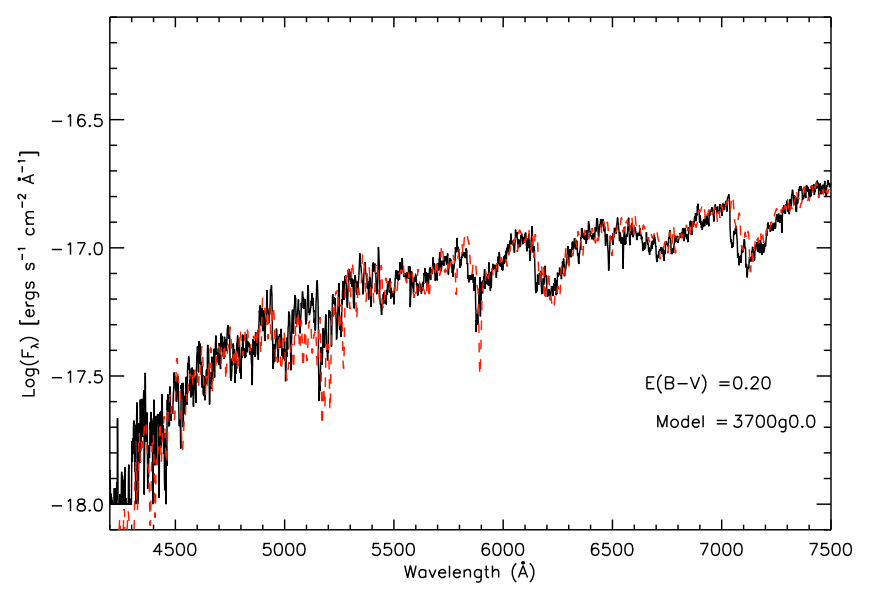

Figure 6. Model Fit. A spectrum of J004330.06+405258.4 (black) is compared with a $3700 \mathrm{~K}, \log g=0.0$ MARCS model from Massey et al. (2009).

\section{DISCUSSION}

We have established that J004330.06+405258.4 is runaway RSG, the first such star clearly identified in another galaxy. Furthermore, with a peculiar radial velocity of $-300 \mathrm{~km} \mathrm{~s}^{-1}$, it is the fastest known runaway massive star of which we are aware. Given its distance, a direct measurement of its tangental component via proper motions is not practical given current methods, but the spatial separation from M31's disk (Figure 3) suggests that the tangential component of the velocity is similar, and thus that the peculiar space velocity is $400-450 \mathrm{~km} \mathrm{~s}^{-1}$.

Lower mass "hypervelocity" stars are known in our own Galaxy; these are stars that are moving at peculiar velocities of $>500 \mathrm{~km} \mathrm{~s}^{-1}$, and, because of their much greater ages, have traveled many tens of $\mathrm{kpc}$ in their lifetimes (see, e.g., Brown et al. 2007). They are escaping from the Galaxy. Our M31 RSG runaway may be a high-mass analog of such stars, rather than related to traditional runaways, given that its velocity is so much greater than the $30 \mathrm{~km} \mathrm{~s}^{-1}$ usually used to distinguish an OB runaway (Blaauw 1961). We note that the peculiar velocities of the other known Galactic RSGs are quite modest by comparison: IRC-10414 at $70 \mathrm{~km} \mathrm{~s}^{-1}$ (Gvaramadze et al. 2014), $\mu$ Cep at $22 \mathrm{~km} \mathrm{~s}^{-1}$ (Cox et al. 2012), and Betelgeuse at $56 \mathrm{~km} \mathrm{~s}^{-1}$ (Noriega-Crespo et al. 1997a). Similarly, only two O-type stars in the classic Cruz-González et al. (1974) catalog have peculiar radial velocities $>100 \mathrm{~km} \mathrm{~s}^{-1}$, and both are under $120 \mathrm{~km} \mathrm{~s}^{-1}$.

We were curious what evidence there was of similar objects in other galaxies. In their study of YSGs in the SMC, Neugent et al. (2010) do, in fact, note that one star, J01020100-7122208, has an anomalously high radial velocity, $+307 \mathrm{~km} \mathrm{~s}^{-1}$, compared to the SMC's systemic velocity of $158 \mathrm{~km} \mathrm{~s}^{-1}$. They suggest the star is either a binary or a runaway; based on a single observation, they cannot tell, but it is clear from their Figure 7 that the star's radial velocity stands out by $100 \mathrm{~km} \mathrm{~s}^{-1}$ from the other YSGs. Similarly, the histograms of the radial velocities of yellow and red supergiants in the LMC studied by Neugent et al. (2012) also show two interesting outliers (their Figure 4): there is a YSG, J04530398-6937285, that has a radial velocity of $+373 \mathrm{~km} \mathrm{~s}^{-1}$, and a RSG, J04482407-7104012 (CPD-71 ${ }^{\circ} 285$ ), with a radial velocity of $+401 \mathrm{~km} \mathrm{~s}^{-1}$, both of which can be compared to the LMC's systemic velocity of $+278 \mathrm{~km} \mathrm{~s}^{-1}$. Again, further observations would be needed to establish that 
these velocities are not high due to binary motion. Among the RSGs in M33, there is an intriguing example, J013403.34 +302611.7 , with a radial velocity that is discrepant by $160 \mathrm{~km} \mathrm{~s}^{-1}$ (see Figure 8 in Drout et al. 2012). None of these are, of course, as extreme as the case of our M31 RSG runaway.

Could J004330.06+405258.4 be a lower mass object? Any alternative explanation needs to account not only for the star's radial velocity but also its photometry. We can essentially rule out the star being a foreground dwarf: an M2 Galactic dwarf at $V=19.2$ would have to be at $0.7 \mathrm{kpc}$ to be this faint, and with a radial velocity of $-630 \mathrm{~km} \mathrm{~s}^{-1}$ it would have to be a hypervelocity star; an unlikely coincidence given how close it is in the plane of the sky to M31's disk. (We would also not expect it to be as reddened.) What if it were an asymptotic giant branch (AGB) star in M31's halo? The bolometric magnitude $M_{\mathrm{bol}}=-7.2\left(\log L / L_{\odot}=4.76\right)$ is faint enough that we expect some contamination by intermediate-mass AGBs in this magnitude range. However, the typical velocity dispersion of halo stars in our own Milky Way is about $100 \mathrm{~km} \mathrm{~s}^{-1}$ (Brown et al. 2010), and we expect that M31's is not much different. We would then still be left trying to explain why the star had a radial velocity $330 \mathrm{~km} \mathrm{~s}^{-1}$ more negative than M31's $-300 \mathrm{~km} \mathrm{~s}^{-1}$ systemic velocity, as this is far in excess of what we expect for a typical halo object.

J004330.06+405258.4 is seen in relatively close proximity (in projection) to the galaxy M32, the compact low-luminosity elliptical visible $\sim 8^{\prime}$ to the west in Figure 3 . Could there be a connection? It is well known that dwarf ellipticals have very little or no current star formation. In the case of M32, about 3\% of its population consists of "young" (ages $<2$ Gyr) metal-rich stars (Monachesi et al. 2012), but there is no evidence of any massive stars. Nor is it likely that J004330.06+405258.4 is an AGB associated with M32, as the galaxy's radial velocity is $-205 \mathrm{~km} \mathrm{~s}^{-1}$ (de Vaucouleurs et al. 1991), which would leave us an even greater discrepancy in radial velocity.

Is J004330.06+405258.4 escaping from M31? This question is largely moot, as the star will only live another million years or so before undergoing an $\mathrm{SN}$ explosion; in that time it will move only another $400-450 \mathrm{pc}$. We do note, however, that it is moving sufficiently fast to have escaped the gravitational attraction of the disk. Although the gravitational well of M31 is complex, with a massive dark-matter halo, we can make a crude estimate simply using the mass of the disk. Rubin \& Ford (1970) calculate that the mass within $24 \mathrm{kpc}$ of the center of M31 is $\sim 2 \times 10^{11} M_{\odot}$. The projected distance of J004330.06 +405258.4 is $5.6 \mathrm{kpc}$. We would thus expect naively that the escape velocity is on the order of $560 \mathrm{~km} \mathrm{~s}^{-1}$. (We note as a reality check that the escape velocity for the Sun from the Milky Way is $550 \mathrm{~km} \mathrm{~s}^{-1}$ according to Smith et al. 2007.) Thus it is not unreasonable that J004330.06+405258.4 has gotten to where it is today.

The presence of bow shocks in association with runaway Galactic RSGs (Mackey et al. 2012) leaves a question as to whether or not we could detect such a structure around our M31 runaway. According to Noriega-Crespo et al. (1997a), Betelgeuse has a bow shock $0.8 \mathrm{pc}$ in size. At the distance of M31, a similiar-sized bow shock would extend about 0 ". 2 . This size is large enough to be readily detectable, at least using space-based imaging. However, it remains to be seen what the effect of our runaway's increased speed and the likely decreased hydrogen column density would be on bow shock size and presence.
We are grateful to the Steward Observatory Time Allocation Committee for their generous allocation of observing time on the MMT, and to Perry Berlind, Mike Calkins, and Marc Lacasse for their excellent support of Hectospec. Nelson Caldwell kindly provided the calibration files for flux calibrating our data, as well as managing the difficult task of queue scheduling our program. Kathryn Neugent helped take some of these observations as part of another project, and also provided very useful scientific suggestions on this project. Emily Levesque and Joe Llama provided useful help with some of the coding for fitting the MARCS models. An anonymous referee kindly made useful suggestions which improved this paper. This publication makes use of data products from the Two Micron All Sky Survey, which is a joint project of the University of Massachusetts and the Infrared Processing and Analysis Center/California Institute of Technology, funded by the National Aeronautics and Space Administration and the National Science Foundation (NSF). K.A.E.'s work was supported through the NSF's Research Experiences for Undergraduates program through Northern Arizona University and Lowell Observatory (AST-1461200) and P.M.'s were partially supported by the NSF through AST-1008020 and through Lowell Observatory. A grant from the Mt. Cuba Astronomical Foundation for computing facilities is gratefully acknowledged.

\section{Facility: MMT (Hectospec).}

\section{REFERENCES}

Blaauw, A. 1961, BAN, 15, 265

Brown, W. R., Geller, M. J., Kenyon, S. J., \& Diaferio, A. 2010, AJ, 139, 59 Brown, W. R., Geller, M. J., Kenyon, S. J., Kurtz, M. J., \& Bromley, B. C. 2007, ApJ, 671, 1708

Cox, N. L. J., Kerschbaum, F., van Marle, A.-J., et al. 2012, A\&A, 537, A35 Cruz-González, C., Recillas-Cruz, E., Costero, R., Peimbert, M., \& Torres-Peimbert, S. 1974, RMxAA, 1, 211

Cutri, R. M., Skrutskie, M. F., van Dyk, S., et al. 2003, yCat, 2246, 0

de Vaucouleurs, G., de Vaucouleurs, A., Corwin, H. G., Jr., et al. 1991, Third Reference Catalogue of Bright Galaxies (New York: Springer)

Drout, M. R., Massey, P., \& Meynet, G. 2012, ApJ, 750, 97

Drout, M. R., Massey, P., Meynet, G., Tokarz, S., \& Caldwell, N. 2009, ApJ, 703, 441

Ekström, S., Georgy, C., Eggenberger, P., et al. 2012, A\&A, 537, A146

Fabricant, D., Fata, R., Roll, J., et al. 2005, PASP, 117, 1411

Fabricant, D. G., Kurtz, M. J., Geller, M. J., et al. 2008, PASP, 120, 1222

Fujii, M. S., \& Portegies Zwart, S. 2011, Sci, 334, 1380

Gies, D. R., \& Bolton, C. T. 1986, ApJS, 61, 419

Gustafsson, B., Edvardsson, B., Eriksson, K., et al. 2008, A\&A, 486, 951

Gvaramadze, V. V., Menten, K. M., Kniazev, A. Y., et al. 2014, MNRAS, 437,843

Lada, C. J., \& Lada, E. A. 2003, ARA\&A, 41, 57

Levesque, E. M., Massey, P., Olsen, K. A. G., et al. 2005, ApJ, 628, 973

Mackey, J., Mohamed, S., Neilson, H. R., Langer, N., \& Meyer, D. M.-A. 2012, ApJL, 751, L10

Massey, P., Olsen, K. A. G., Hodge, P. W., et al. 2006, AJ, 131, 2478

Massey, P., Olsen, K. A. G., Hodge, P. W., et al. 2007, AJ, 133, 2393

Massey, P., Plez, B., Levesque, E. M., et al. 2005, ApJ, 634, 1286

Massey, P., Silva, D. R., Levesque, E. M., et al. 2009, ApJ, 703, 420

Monachesi, A., Trager, S. C., Lauer, T. R., et al. 2012, ApJ, 745, 97

Neugent, K. F., Massey, P., Skiff, B., et al. 2010, ApJ, 719, 1784

Neugent, K. F., Massey, P., Skiff, B., \& Meynet, G. 2012, ApJ, 749, 177

Noriega-Crespo, A., van Buren, D., Cao, Y., \& Dgani, R. 1997a, AJ, 114, 837

Noriega-Crespo, A., van Buren, D., \& Dgani, R. 1997b, AJ, 113, 780

Oh, S., Kroupa, P., \& Pflamm-Altenburg, J. 2015, ApJ, 805, 92

Rubin, V. C., \& Ford, W. K., Jr. 1970, ApJ, 159, 379

Schlegel, D. J., Finkbeiner, D. P., \& Davis, M. 1998, ApJ, 500, 525

Smith, M. C., Ruchti, G. R., Helmi, A., et al. 2007, MNRAS, 379, 755

van den Bergh, S. 2000, The Galaxies of the Local Group (Cambridge:

Cambridge Univ. Press) 